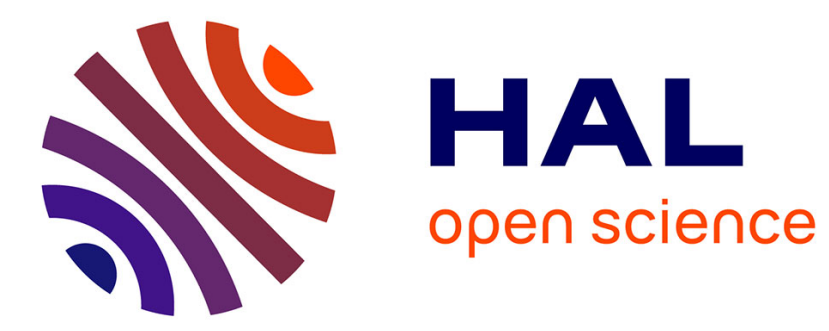

\title{
Theoretical analysis of the zigzag instability of a vertical co-rotating vortex pair in a strongly stratified fluid Pantxika Otheguy, Paul Billant, Jean-Marc Chomaz
}

\section{To cite this version:}

Pantxika Otheguy, Paul Billant, Jean-Marc Chomaz. Theoretical analysis of the zigzag instability of a vertical co-rotating vortex pair in a strongly stratified fluid. Journal of Fluid Mechanics, 2007, 584 (august), pp.103-123. 10.1017/s0022112007006295 . hal-01023342

\section{HAL Id: hal-01023342}

https://hal-polytechnique.archives-ouvertes.fr/hal-01023342

Submitted on 20 Jul 2014

HAL is a multi-disciplinary open access archive for the deposit and dissemination of scientific research documents, whether they are published or not. The documents may come from teaching and research institutions in France or abroad, or from public or private research centers.
L'archive ouverte pluridisciplinaire HAL, est destinée au dépôt et à la diffusion de documents scientifiques de niveau recherche, publiés ou non, émanant des établissements d'enseignement et de recherche français ou étrangers, des laboratoires publics ou privés. 


\title{
Theoretical analysis of the zigzag instability of a vertical co-rotating vortex pair in a strongly stratified fluid
}

\author{
PANTXIKA OTHEGUY†,PAUL BILLANT \\ AND JEAN-MARC CHOMAZ \\ LadHyX, CNRS, École Polytechnique, F-91128 Palaiseau Cedex, France
}

(Received 31 January 2006 and in revised form 25 February)

A long-wavelength stability analysis of two co-rotating Gaussian vertical vortices in an inviscid strongly stratified fluid is conducted for vortices separated by a large distance $b$ compared to their radius $a(b \gg a)$. This analysis predicts and explains the zigzag instability found by a numerical stability analysis in a companion paper (Otheguy, Chomaz \& Billant, J. Fluid. Mech. vol. 553, 2006, p. 253). The zigzag instability results from the coupling between the bending perturbations of each vortex and the external strain that one vortex induces on the other $S=\Gamma / 2 \pi b^{2}$, where $\Gamma$ is the circulation of the vortices. The analysis predicts that the maximum growth rate of the instability is twice the strain $S$ and that the most unstable vertical wavelength $\lambda$ scales as the buoyancy length, defined by $L_{B}=\Gamma / \pi a N$, multiplied by the ratio $b / a$, i.e. $\lambda \propto F_{h} b$, where $F_{h}=\Gamma / \pi a^{2} N$ is the horizontal Froude number. The asymptotic results are in very good agreement with the numerical results.

\section{Introduction}

In stably and strongly stratified flows, the velocity field is mainly horizontal because vertical displacements are inhibited by the buoyancy force due to the vertical stratification (Riley, Metcalfe \& Weissman 1981; Lilly 1983). Two kind of motions remain possible: internal waves and horizontal vortical motions. Many studies have shown that the latter motions take the form of layers or pancake vortices with a much larger length scale in the horizontal direction than in the vertical one (Herring \& Métais 1989; Park, Whitehead \& Gnanadeskian 1994; Fincham, Maxworthy \& Spedding 1996; Spedding, Browand \& Fincham 1996; Holford \& Linden 1999; Riley \& Lelong 2000; Bonnier, Eiff \& Bonneton 2000; Billant \& Chomaz 2000a; Godeferd \& Staquet 2003; Waite \& Bartello 2004; Praud, Fincham \& Sommeria 2005 among others). Of particular interest is the vertical length scale that characterizes these vortices and these horizontal layers.

At present, two regimes have been identified for the selection of the vertical length scale. When viscous effects due to vertical shearing of horizontal motions are dominant over vertical transport terms (Godoy-Diana, Chomaz \& Billant 2004), the vertical length scale is determined by viscous effects independently of the stratification as

$\dagger$ Present address: Centre Technique Littoral, Lyonnaise des Eaux, Pavillon Izarbel, Technopole Izarbel, 64210 Bidart, France. 
shown by the experiments by Spedding et al. (1996), Fincham et al. (1996), Bonnier et al. (2000) and Praud et al. (2005).

On the other hand, when viscous effects due to vertical shearing of horizontal motions are small compared to vertical transport terms, stratified flows experiments (Park et al. 1994; Holford \& Linden 1999; Billant \& Chomaz 2000a) and numerical simulations (Waite \& Bartello 2004; Lindborg 2006) exhibit layers with a thickness proportional to the buoyancy length $U / N$, where $U$ is the characteristic horizontal velocity scale and $N$ the Brunt-Väisälä frequency. Billant \& Chomaz (2001) have shown that this scaling law is universal for all strongly stratified inviscid flows initially vertically coherent. For this small vertical length scale, the vertical transport is not negligible even though the vertical velocity is small so that the dynamics is not two-dimensional.

In the particular case of a vortex dipole, i.e. two counter-rotating vertical vortices, in a stratified fluid, Billant \& Chomaz $(2000 a-c)$ have described a three-dimensional instability, the zigzag instability, which explains how a vertically coherent stratified flow can spontaneously develop layers with a thickness of the order of the buoyancy length scale.

In order to show that the zigzag instability is a generic mechanism for the formation of layers, not restricted to the particular case of a vortex dipole, we have investigated numerically in a companion paper (Otheguy, Chomaz \& Billant 2006) the linear stability of a vortex couple, i.e. a pair of co-rotating vertical vortices in a stratified fluid. We have shown that the zigzag instability also affects such a flow. Its growth rate scales as the external strain rate $S=\Gamma / 2 \pi b^{2}$ (where $\Gamma$ is the circulation of the vortices and $b$ the separation distance between the vortices) that each vortex induces on the other and that the most unstable wavelength $\lambda$ scales on the buoyancy length (defined here as $L_{B}=\Gamma / \pi a N$ ) multiplied by the ratio $b / a$, i.e. $\lambda \propto F_{h} b$ (where $F_{h}=\Gamma / \pi a^{2} N$ is the horizontal Froude number and $a$ the radius of the vortices).

In the present paper, we explain the physical mechanism of the zigzag instability and the scaling laws for its growth rate and wavelength by investigating theoretically the stability of two co-rotating vertical Gaussian vortices (Lamb-Oseen vortices, Lamb 1932; Saffman 1992) in a strongly stratified fluid. In the two-dimensional case, the vortices rotate around each other at a constant angular velocity $\Omega=\Gamma / \pi b^{2}$. Because of rotational invariance, this basic flow is neutral to the phase perturbation corresponding to a small variation of the reference angle of the line joining the vortex centres. It is also neutral to a small variation of the separation distance $b$ between the vortices because of the invariance derived from the parameter $b$ describing continuously the family of vortex pair solutions.

In the spirit of the analysis of Billant \& Chomaz (2000b) for a pair of counterrotating vortices (a Lamb-Chaplygin vortex pair) in a stratified fluid, we shall demonstrate that the three-dimensional destabilization of these two phase modes leads to the zigzag instability. The approach is based on an asymptotic analysis in the limit of small Froude numbers (horizontal $F_{h}$ and vertical $F_{v}=\Gamma / \pi a L_{v} N$, where $L_{v}$ is the vertical length scale) and small ratio $a / b$.

The assumption that $F_{h}$ is small means that the flow is strongly stratified: the time scale of the horizontal motion is large compared to the Brunt-Väisälä period. The hypothesis that $F_{v}$ is small corresponds to the assumption of a vertical wavelength large compared to the buoyancy length. We also assume that $a / b$ is small because an exact solution of the two-dimensional Euler equation describing a co-rotating vortex pair is not known. The basic state is therefore computed asymptotically for vortices separated by a large distance $b$ compared to their radius $a$. 
The final result of this asymptotic analysis is two coupled equations for the two phase variables $(\delta \alpha(z, t), \delta b(z, t))$, in dimensional form,

$$
\begin{gathered}
\frac{\partial \delta \alpha}{\partial t}=-\frac{2 \Gamma}{\pi b^{3}} \delta b+\frac{\Gamma}{\pi b} D_{0} F_{h}^{2} \frac{\partial^{2} \delta b}{\partial z^{2}}, \\
\frac{\partial \delta b}{\partial t}=-\frac{\Gamma b}{\pi} D_{0} F_{h}^{2} \frac{\partial^{2} \delta \alpha}{\partial z^{2}},
\end{gathered}
$$

where $\delta \alpha(z, t)$ is the perturbation of the reference angle of the vortex pair, $\delta b(z, t)$ the perturbation of the distance separating the two vortices and $D_{0}=(7 / 8) \ln 2-(9 / 16)$ $\ln 3$ is a coefficient computed from the asymptotics. In the two-dimensional limit $(\partial / \partial z=0)$, these equations reduce to

$$
\begin{gathered}
\frac{\partial \delta \alpha}{\partial t}=-\frac{2 \Gamma}{\pi b^{3}} \delta b, \\
\frac{\partial \delta b}{\partial t}=0 .
\end{gathered}
$$

The first equation (1.3) is derived simply from perturbing $(\alpha \rightarrow \alpha+\delta \alpha, b \rightarrow b+\delta b)$ the equation governing the rotation of the vortex pair in the laboratory frame of reference,

$$
\frac{\partial \alpha}{\partial t}=\frac{\Gamma}{\pi b^{2}}
$$

while equation (1.4) tells us that $\delta b$ does not evolve because of momentum conservation. Physically, if the separation distance is slightly decreased $b \rightarrow b+\delta b$ (with $\delta b<0$ ), equation (1.3) implies that the perturbation angle $\delta \alpha$ increases algebraically with time, $\delta \alpha=\delta \Omega t$ with $\delta \Omega=-\left(2 \Gamma / \pi b^{3}\right) \delta b$. This is because the vortex pair is no longer steady in the reference frame rotating at rate $\Omega=\Gamma / \pi b^{2}$ but rotates at rate $\delta \Omega$ owing to the slight decrease of the separation distance $\delta b$.

When $\partial / \partial z \neq 0$, equations (1.1)-(1.2) show that weak three-dimensional effects couple the two phase variables and we shall see that this coupling is unstable and leads to the zigzag instability.

The paper is organized as follows. The stability problem is presented in $\S 2$. The asymptotic analysis is carried out in $\S 3$. The stability of the two phase equations is analysed in $\S 4$. In $\S 5$, the asymptotic results are shown to be in very good agreement with the numerical results of Otheguy et al. (2006).

\section{Stability problem}

The flow is assumed to satisfy the Boussinesq approximation of the Euler equation, and to be incompressible and inviscid with a non-diffusive stratifying agent:

$$
\begin{gathered}
\frac{\mathrm{D} \boldsymbol{u}}{\mathrm{D} t}=-\frac{1}{\rho_{r}} \nabla p-g \frac{\rho}{\rho_{r}} \boldsymbol{e}_{z}, \\
\nabla \cdot \boldsymbol{u}=0, \\
\frac{\mathrm{D} \rho_{t}}{\mathrm{D} t}=0,
\end{gathered}
$$

where $\boldsymbol{u}=\left(u_{x}, u_{y}, u_{z}\right)$ is the velocity vector in Cartesian coordinates $(x, y, z), p$ the pressure, $g$ the acceleration due to gravity, $\boldsymbol{e}_{z}$ the unit vector pointing upward, and $\rho_{t}$ 


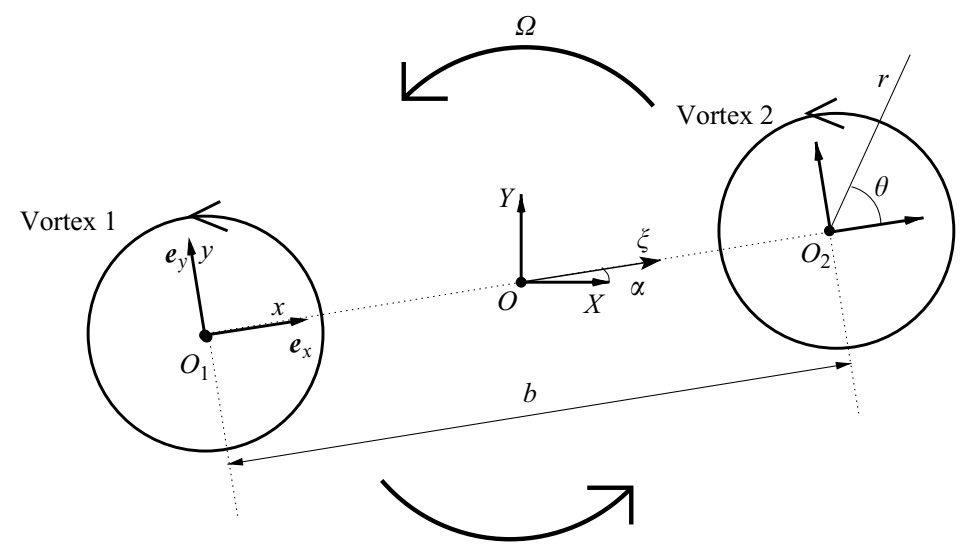

FIGURE 1. Sketch of the two co-rotating vortices with the different coordinate systems used.

the total density of the flow expressed as the sum of a constant reference density $\rho_{r}$, a linear mean density profile $\bar{\rho}(z)$ and the perturbation density $\rho(\boldsymbol{x}, t)$ :

$$
\rho_{t}(\boldsymbol{x}, t)=\rho_{r}+\bar{\rho}(z)+\rho(\boldsymbol{x}, t) .
$$

\subsection{Basic state}

We consider as basic state a pair of co-rotating vertical vortices separated by a distance $b$ (figure 1) and where as a first approximation each vortex is a Lamb-Oseen vortex (Lamb 1932; Saffman 1992) of circulation $\Gamma$ and radius $a$. In the following, horizontal length and time are non-dimensionalized by $a$ and $\tau=\pi a^{2} / \Gamma$ respectively. Thus, the non-dimensional separation distance is $\Lambda=b / a$ and the non-dimensional vertical vorticity of each vortex (for example vortex 2 ) is

$$
\omega=\mathrm{e}^{-r^{2}}
$$

where $r$ is the non-dimensional radial distance in the local cylindrical coordinates $(r$, $\theta$ ) with origin at the centre of vortex 2 (figure 1). The corresponding velocity field of the second vortex is

$$
\boldsymbol{U}=v_{\theta}(r) \boldsymbol{e}_{\theta}
$$

where

$$
v_{\theta}=\frac{1}{2 r}\left(1-\mathrm{e}^{-r^{2}}\right)
$$

and $\boldsymbol{e}_{\theta}$ is the azimuthal unit vector. A single Lamb-Oseen vortex is an exact solution of the two-dimensional Euler equation, but the sum of two vortices is no longer an exact solution because each vortex feels the non-uniform velocity field induced by the other. However, if the separation distance is large compared to the vortex radius (if $\Lambda$ is large), the velocity field induced by vortex 2 on vortex 1 is at leading order

$$
U_{2}=-\frac{1}{2 \Lambda} \boldsymbol{e}_{y}-\frac{1}{2 \Lambda^{2}}\left[x \boldsymbol{e}_{y}+y \boldsymbol{e}_{x}\right]+O\left(\frac{x^{2}}{\Lambda^{3}}, \frac{y^{2}}{\Lambda^{3}}, \frac{x y}{\Lambda^{3}}\right)
$$

where $(x, y)$ are the local Cartesian coordinates with origin at the centre of vortex 1 and with the unit vector $\boldsymbol{e}_{x}$ aligned along the line joining the two vortex centres (figure 1). At leading order, vortex 1 is therefore advected at the constant velocity $-1 / 2 \Lambda \boldsymbol{e}_{y}$ and since the two vortices are chosen with equal circulation, vortex 2 is 
advected at velocity $1 / 2 \Lambda \boldsymbol{e}_{y}$ by vortex 1 . Thus the vortex pair rotates uniformly at the angular velocity $\Omega=1 / \Lambda^{2}$ around the middle point $O$ between the two vortex centres. Therefore, at leading order in $1 / \Lambda$, a vortex pair consisting of two axisymmetric Gaussian vortices is a steady solution of the Euler equation in the frame of reference rotating at the angular velocity $\Omega$. At next order, $O\left(1 / \Lambda^{2}\right)$, the velocity field exerted by the companion vortex is non-uniform: $-1 / 2 \Lambda^{2}\left(x \boldsymbol{e}_{y}+y \boldsymbol{e}_{x}\right)$, and corresponds to a uniform external strain field $S=1 / 2 \Lambda^{2}$. Moore \& Saffman (1975), Moffatt, Kida \& Ohkitani (1994), Eloy \& Le Dizès (1999) and Le Dizès \& Laporte (2002) have shown that an axisymmetric vortex under the influence of such a uniform strain field is deformed elliptically but remains a steady solution of the Euler equation. The interaction between strain and vorticity leads to an amplification of the strain in the vortex core (the so-called internal strain rate) but the core vorticity is not modified. In the case of a Gaussian vortex in a non-rotating reference frame, the internal strain rate is 2.52 times the external strain $S=1 / 2 \Lambda^{2}$ (Eloy \& Le Dizès 1999).

We shall not describe further the order $1 / \Lambda^{2}$ because its detailed solution will not be necessary in the following analysis. The important point to remember is that two co-rotating Gaussian vortices separated by a sufficiently large distance $\Lambda$ adapt to each other to give a steady basic state in the reference frame rotating with the vortex pair at the angular velocity $\Omega=1 / \Lambda^{2}$. In this frame, the velocity field of the basic state can be written

$$
\boldsymbol{U}_{b}=\boldsymbol{U}_{b 1}+\boldsymbol{U}_{b 2}-\frac{\xi}{\Lambda^{2}} \boldsymbol{e}_{\alpha}
$$

where the last term is the velocity due to the rotation of the reference frame, with ( $\xi$, $\alpha$ ) the cylindrical coordinates centred on $O$ (figure 1). $\boldsymbol{U}_{b i}$ corresponds to the velocity field of vortex $i(i=1,2)$ adapted to the strain of the other vortex, i.e.

$$
\boldsymbol{U}_{b i}=\boldsymbol{U}_{a i}+\frac{1}{\Lambda^{2}} \boldsymbol{U}_{e i}
$$

where $\boldsymbol{U}_{a i}$ is the axisymmetric velocity field of a single Lamb-Oseen vortex as given by (2.4) and $\boldsymbol{U}_{e i}$ is the velocity due to the adaptation of this vortex to the strain induced by the companion vortex. The latter velocity vanishes rapidly outside the vortex core (Le Dizès \& Laporte 2002; Eloy \& Le Dizès 1999), and we shall see that it plays no role in the zigzag instability, in contrast to the elliptic instability.

Since the basic flow $\boldsymbol{U}_{b}$ is two-dimensional, it is convenient to introduce a streamfunction $\psi_{b}$ such that $\boldsymbol{U}_{b}=-\nabla \times\left(\psi_{b} \boldsymbol{e}_{z}\right)$. Even though its exact expression is not known, the family of basic flows considered in the present paper can be therefore written formally

$$
\psi_{b}(\xi, \alpha ; \Lambda)
$$

where the separation distance $\Lambda$ is a parameter describing continuously the family of solutions. This basic flow is steady and therefore satisfies the two-dimensional vertical vorticity equation

$$
J\left(\psi_{b}, \Delta \psi_{b}\right)=\frac{1}{\xi} \frac{\partial \psi_{b}}{\partial \xi} \frac{\partial \Delta \psi_{b}}{\partial \alpha}-\frac{1}{\xi} \frac{\partial \psi_{b}}{\partial \alpha} \frac{\partial \Delta \psi_{b}}{\partial \xi}=0
$$

where $J$ denotes the Jacobian and $\Delta$ the Laplacian.

\subsection{Scaling analysis}

The scaling analysis of Riley et al. (1981) for stratified vortical flows when buoyancy effects are dominant is used to non-dimensionalize the governing equations (2.1) in 
the reference frame rotating at rate $\Omega$ :

$$
\begin{gathered}
\frac{\partial \boldsymbol{u}_{h}}{\partial t}+\left[\nabla_{h} \boldsymbol{u}_{h}\right] \cdot \boldsymbol{u}_{h}+F_{v}^{2} u_{z} \frac{\partial \boldsymbol{u}_{h}}{\partial z}+2 \Omega \boldsymbol{e}_{z} \times \boldsymbol{u}_{h}=-\nabla_{h} p, \\
F_{h}^{2}\left(\frac{\partial u_{z}}{\partial t}+\left[\nabla_{h} u_{z}\right] \cdot \boldsymbol{u}_{h}+F_{v}^{2} u_{z} \frac{\partial u_{z}}{\partial z}\right)=-\frac{\partial p}{\partial z}-\rho, \\
\nabla_{h} \cdot \boldsymbol{u}_{h}+F_{v}^{2} \frac{\partial u_{z}}{\partial z}=0, \\
\frac{\partial \rho}{\partial t}+\left[\nabla_{h} \rho\right] \cdot \boldsymbol{u}_{h}+F_{v}^{2} u_{z} \frac{\partial \rho}{\partial z}-u_{z}=0,
\end{gathered}
$$

where $\boldsymbol{u}_{h}$ and $\nabla_{h}$ are the horizontal components of $\boldsymbol{u}$ and $\nabla, F_{h}=\Gamma / \pi a^{2} N$ the horizontal Froude number with $N=\sqrt{-\left(g / \rho_{r}\right) \mathrm{d} \bar{\rho} / \mathrm{d} z}$ the Brunt-Väisälä frequency and $F_{v}=\Gamma / \pi a L_{v} N$ the vertical Froude number. The same notation has been kept for non-dimensional variables. Time has been non-dimensionalized by the inverse of the maximum vorticity of the basic state $\tau=\pi a^{2} / \Gamma$, and horizontal length by the vortex radius $a$. The vertical length is non-dimensionalized by $L_{v}$ (at present arbitrary), horizontal velocity by $\Gamma / \pi a$, vertical velocity by $F_{h} F_{v} \Gamma / \pi a$, pressure by $\rho_{r}(\Gamma / \pi a)^{2}$ and density perturbation by $\rho_{r} \Gamma^{2} / g \pi^{2} a^{2} L_{v}$. It is important to note that $F_{h}$ and $F_{v}$ can be small independently.

In the limit $F_{h}=0$, the vertical momentum equation $(2.11 b)$ reduces to the hydrostatic equilibrium. In the limit $F_{v}=0$, the equations $(2.11 a, c)$ become the twodimensional incompressible Euler equations.

\subsection{Linearized equations}

The basic flow (2.7) is subjected to infinitesimal perturbations, denoted by a tilde:

$$
\left(\boldsymbol{u}_{h}, u_{z}, p, \rho\right)=\left(\boldsymbol{U}_{b}, 0, p_{b}, 0\right)+\left(\tilde{\boldsymbol{u}}_{h}, \tilde{u}_{z}, \tilde{p}, \tilde{\rho}\right),
$$

and equations (2.11) are linearized to obtain the equations governing the perturbations:

$$
\begin{gathered}
\frac{\partial \tilde{\boldsymbol{u}}_{h}}{\partial t}+\left[\nabla_{h} \tilde{\boldsymbol{u}}_{h}\right] \cdot \boldsymbol{U}_{b}+\left[\nabla_{h} \boldsymbol{U}_{b}\right] \cdot \tilde{\boldsymbol{u}}_{h}+2 \Omega \boldsymbol{e}_{z} \times \tilde{\boldsymbol{u}}_{h}=-\nabla_{h} \tilde{p}, \\
F_{h}^{2}\left(\frac{\partial \tilde{u}_{z}}{\partial t}+\left[\nabla_{h} \tilde{u}_{z}\right] \cdot \boldsymbol{U}_{b}\right)=-\frac{\partial \tilde{p}}{\partial z}-\tilde{\rho}, \\
\nabla_{h} \cdot \tilde{\boldsymbol{u}}_{h}+F_{v}^{2} \frac{\partial \tilde{u}_{z}}{\partial z}=0, \\
\frac{\partial \tilde{\rho}}{\partial t}+\left[\nabla_{h} \tilde{\rho}\right] \cdot \boldsymbol{U}_{b}-\tilde{u}_{z}=0 .
\end{gathered}
$$

\section{Asymptotic problem}

3.1. Formulation of the asymptotic problem

In order to solve asymptotically the linearized equations (2.13), we write the perturbation as an expansion in the vertical Froude number $F_{v}$ :

$$
(\tilde{\boldsymbol{u}}, \tilde{p}, \tilde{\rho})=\left(\tilde{\boldsymbol{u}}_{0}, \tilde{p}_{0}, \tilde{\rho}_{0}\right)+F_{v}{ }^{2}\left(\tilde{\boldsymbol{u}}_{2}, \tilde{p}_{2}, \tilde{\rho}_{2}\right)+\cdots,
$$


and each order is further expanded with the small parameter $1 / \Lambda^{2}$ :

$$
\left.\begin{array}{l}
\left(\tilde{\boldsymbol{u}}_{0}, \tilde{p}_{0}, \tilde{\rho}_{0}\right)=\left(\tilde{\boldsymbol{u}}_{00}, \tilde{p}_{00}, \tilde{\rho}_{00}\right)+\frac{1}{\Lambda^{2}}\left(\tilde{\boldsymbol{u}}_{02}, \tilde{p}_{02}, \tilde{\rho}_{02}\right)+\cdots, \\
\left(\tilde{\boldsymbol{u}}_{2}, \tilde{p}_{2}, \tilde{\rho}_{2}\right)=\left(\tilde{\boldsymbol{u}}_{20}, \tilde{p}_{20}, \tilde{\rho}_{20}\right)+\frac{1}{\Lambda^{2}}\left(\tilde{\boldsymbol{u}}_{22}, \tilde{p}_{22}, \tilde{\rho}_{22}\right)+\cdots,
\end{array}\right\}
$$

because the basic state is known only as an expansion in $1 / \Lambda$. We shall see that the zeroth order (subscript 00), and orders $F_{v}{ }^{2}$ (subscript 02 ) and $1 / \Lambda^{2}$ (subscript 20) are sufficient to describe the instability at leading order and therefore the expansion can be written:

$(\tilde{\boldsymbol{u}}, \tilde{p}, \tilde{\rho})=\left(\tilde{\boldsymbol{u}}_{00}, \tilde{p}_{00}, \tilde{\rho}_{00}\right)+\frac{1}{\Lambda^{2}}\left(\tilde{\boldsymbol{u}}_{02}, \tilde{p}_{02}, \tilde{\rho}_{02}\right)+F_{v}{ }^{2}\left(\tilde{\boldsymbol{u}}_{20}, \tilde{p}_{20}, \tilde{\rho}_{20}\right)+O\left(\frac{F_{v}{ }^{2}}{\Lambda^{2}}, F_{v}{ }^{4}, \frac{1}{\Lambda^{4}}\right)$.

As usual in multiple-scale analysis, the time variable is also expanded, introducing slow time scales $t_{02}$ and $t_{20}$ that can be treated as independent variables

$$
\frac{\partial}{\partial t}=\frac{1}{\Lambda^{2}} \frac{\partial}{\partial t_{02}}+F_{v}{ }^{2} \frac{\partial}{\partial t_{20}}+\cdots .
$$

We shall also consider that the horizontal Froude number is small and related to $F_{v}$ by $F_{h}=\delta F_{v}$ where $\delta=L_{v} / a$ is the aspect ratio.

The order of $\delta$ will be assumed to be less than or equal to one. The purpose of this assumption is to avoid a third expansion with the small horizontal Froude number, which would lead to the same result provided $\delta \ll 1 / F_{v}$, i.e. $F_{h} \ll 1$.

\subsection{Order-zero problem in $F_{v}$}

Although the zeroth-order perturbation in $F_{v}$ has only to be determined up to the second order in $1 / \Lambda$, it will be determined exactly in this section without resorting to an expansion with the parameter $1 / \Lambda$. Inserting the expansion (3.1) in the linearized equations (2.13) gives at zeroth order in $F_{v}$

$$
\begin{gathered}
\frac{1}{\Lambda^{2}} \frac{\partial \tilde{\boldsymbol{u}}_{h 0}}{\partial t_{02}}+\left[\nabla_{h} \tilde{\boldsymbol{u}}_{h 0}\right] \cdot \boldsymbol{U}_{b}+\left[\nabla_{h} \boldsymbol{U}_{b}\right] \cdot \tilde{\boldsymbol{u}}_{h 0}+2 \Omega \boldsymbol{e}_{z} \times \tilde{\boldsymbol{u}}_{h 0}=-\nabla_{h} \tilde{p}_{0}, \\
0=-\frac{\partial \tilde{p}_{0}}{\partial z}-\tilde{\rho}_{0}^{\prime}, \\
\nabla_{h} \cdot \tilde{\boldsymbol{u}}_{h 0}=0, \\
\frac{1}{\Lambda^{2}} \frac{\partial \tilde{\rho}_{0}}{\partial t_{02}}+\left[\nabla_{h} \tilde{\rho}_{0}\right] \cdot \boldsymbol{U}_{b}-\tilde{u}_{z 0}=0 .
\end{gathered}
$$

The horizontal momentum equations (3.5a) and (3.5c) do not involve the vertical derivative so that it is convenient to introduce a streamfunction $\tilde{\psi}_{0}\left(x, y, z, t_{02}\right)$ defined by $\tilde{\boldsymbol{u}}_{h 0}=-\nabla \times\left(\tilde{\psi}_{0} \boldsymbol{e}_{z}\right)$ and to use the equation for the vertical vorticity $\Delta_{h} \tilde{\psi}_{0}$ by taking the horizontal curl of $(3.5 a)$

$$
\frac{1}{\Lambda^{2}} \frac{\partial}{\partial t_{02}} \Delta_{h} \tilde{\psi}_{0}+J\left(\psi_{b}, \Delta_{h} \tilde{\psi}_{0}\right)+J\left(\tilde{\psi}_{0}, \Delta_{h} \psi_{b}\right)=0
$$

Solutions of (3.6) may be found following Billant \& Chomaz (2000b) by making use of the invariance of equation (3.6) and the invariance associated with each parameter describing continuously a family of basic states. In the present paper, we focus on the solutions derived from the rotational invariance and from the invariance associated 
with parameter $\Lambda$ describing the family of co-rotating vortex pair solutions. More precisely, we choose the leading-order perturbation as

$$
\tilde{\psi}_{0}=-\delta \alpha \frac{\partial}{\partial \alpha} \psi_{b}+\Lambda \delta \Lambda \frac{\partial}{\partial \Lambda}\left(\psi_{b}+\frac{\xi^{2}}{2 \Lambda^{2}}\right) .
$$

The first term corresponds to the neutral mode derived from the rotational invariance: if $\psi_{b}(\xi, \alpha, \Lambda)$ is a solution, then the same solution but rotated by a constant angle $\delta \alpha, \psi_{b}(\xi, \alpha-\delta \alpha, \Lambda)$, is also an exact solution. For an infinitesimal rotation $\delta \alpha \ll 1$, a Taylor expansion gives the first term of (3.7):

$$
\psi_{b}(\xi, \alpha-\delta \alpha, \Lambda)-\psi_{b}(\xi, \alpha, \Lambda)=-\delta \alpha \frac{\partial \psi_{b}}{\partial \alpha} .
$$

Similarly, the invariance linked to the parameter $\Lambda$ implies that if $\Lambda$ is varied, $\Lambda \rightarrow \Lambda(1+\delta \Lambda) \dagger$ where $\delta \Lambda$ is a constant, $\psi_{b}(\xi, \alpha, \Lambda(1+\delta \Lambda))$ is also an exact solution but in the reference frame rotating at rate $1 / \Lambda^{2}(1+\delta \Lambda)^{2}$. For $\delta \Lambda \ll 1$, a Taylor expansion of the perturbed streamfunction re-expressed in the original frame rotating at rate $1 / \Lambda^{2}$ gives the second term of (3.7):

$$
\psi_{b}(\xi, \alpha, \Lambda(1+\delta \Lambda))+\frac{\xi^{2}}{2 \Lambda^{2}(1+\delta \Lambda)^{2}}-\frac{\xi^{2}}{2 \Lambda^{2}}-\psi_{b}(\xi, \alpha, \Lambda)=\Lambda \delta \Lambda \frac{\partial}{\partial \Lambda}\left(\psi_{b}+\frac{\xi^{2}}{2 \Lambda^{2}}\right) .
$$

As usual in multiple-scale analysis, the phase variables $(\delta \alpha, \delta \Lambda)$ are considered functions of the slow times $\left(t_{02}, t_{20}\right)$ and also of the vertical coordinate $z$ because equation (3.6) does not involve $z$ derivatives. Inserting perturbation (3.7) in (3.6) leads to

$$
-\frac{\partial \delta \alpha}{\partial t_{02}} \frac{\partial \Delta \psi_{b}}{\partial \alpha}+\Lambda \frac{\partial \delta \Lambda}{\partial t_{02}} \frac{\partial \Delta \psi_{b}}{\partial \Lambda}-2 \delta \Lambda \frac{\partial \Delta \psi_{b}}{\partial \alpha}=0,
$$

where we have used the fact that

$$
J\left(\frac{\partial \psi_{b}}{\partial \alpha}, \Delta \psi_{b}\right)+J\left(\psi_{b}, \frac{\partial \Delta \psi_{b}}{\partial \alpha}\right)=0
$$

and

$$
J\left(\frac{\partial \psi_{b}}{\partial \Lambda}, \Delta \psi_{b}\right)+J\left(\psi_{b}, \frac{\partial \Delta \psi_{b}}{\partial \Lambda}\right)=0
$$

since the basic flow $\psi_{b}$ satisfies $J\left(\psi_{b}, \Delta \psi_{b}\right)=0$. The particular perturbation (3.7) is therefore a solution of (3.6) if $(\delta \alpha, \delta \Lambda)$ satisfy the phase equations

$$
\begin{gathered}
\frac{\partial \delta \alpha}{\partial t_{02}}=-2 \delta \Lambda, \\
\frac{\partial \delta \Lambda}{\partial t_{02}}=0 .
\end{gathered}
$$

Note that the explicit expression for $\psi_{b}$ has not been necessary to derive these phase equations. These equations show that the rotational invariance and the invariance linked to the parameter $\Lambda$ are coupled. They simply describe the fact that if the separation distance between the two vortices $\Lambda$ is slightly perturbed, $\Lambda \rightarrow \Lambda(1+\delta \Lambda)$,

$\dagger$ Note that $\delta \Lambda$ corresponds to the relative variation of the non-dimensional separation distance $\Lambda$ in order that $\delta \Lambda \ll 1$ even if $\Lambda$ is large. 


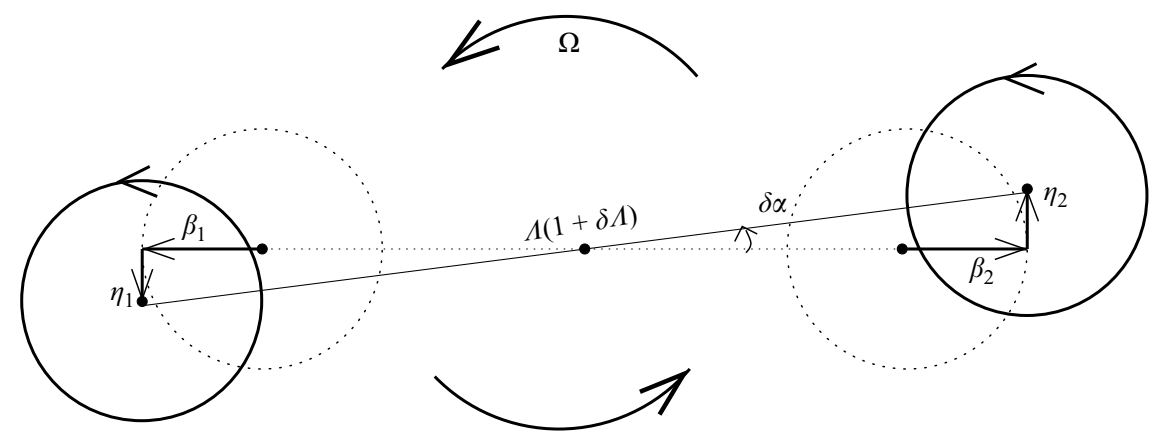

FIGURE 2. The basic vortex pair (dotted line) is perturbed by a small rotation $\delta \alpha$ and a small variation of the separation distance $\Lambda \rightarrow \Lambda(1+\delta \Lambda)$. The perturbed vortex pair is represented by solid lines. Since $\delta \alpha$ and $\delta \Lambda$ are small, the perturbation is equivalent to $x$ - and $y$-translations of the two vortices in opposite directions such that the centres of the perturbed vortices are located at $\left(\beta_{2}=\Lambda \delta \Lambda / 2, \eta_{2}=\Lambda \delta \alpha / 2\right)$ and $\left(\beta_{1}=-\Lambda \delta \Lambda / 2, \eta_{1}=-\Lambda \delta \alpha / 2\right)$.

then the vortex pair will no longer be steady in the original frame of reference rotating at angular velocity $1 / \Lambda^{2}$ but will rotate at velocity

$$
\delta \Omega=\frac{1}{\Lambda^{2}(1+\delta \Lambda)^{2}}-\frac{1}{\Lambda^{2}}=-\frac{2}{\Lambda^{2}} \delta \Lambda .
$$

Using (3.7), we can now determine the pressure $\tilde{p}_{0}$ from $(3.5 a)$ :

$$
\tilde{p}_{0}=-\delta \alpha+\Lambda \delta \Lambda\left[\frac{\partial p_{b}}{\partial \Lambda}-2 \frac{\xi^{2}}{\Lambda^{5}}\right]
$$

where $p_{b}$ is the pressure of the basic state. The density perturbation is then obtained from the hydrostatic balance $(3.5 b)$ :

$$
\tilde{\rho}_{0}=\frac{\partial \delta \alpha}{\partial z} \frac{\partial p_{b}}{\partial \alpha}-\Lambda \frac{\partial \delta \Lambda}{\partial z}\left[\frac{\partial p_{b}}{\partial \Lambda}-2 \frac{\xi^{2}}{\Lambda^{5}}\right]
$$

and the density equation $(3.5 d)$ imposes the vertical velocity

$$
\tilde{u}_{z 0}=\frac{\partial \delta \alpha}{\partial z} J\left(\psi_{b}, \frac{\partial p_{b}}{\partial \alpha}\right)-\Lambda \frac{\partial \delta \Lambda}{\partial z}\left[J\left(\psi_{b}, \frac{\partial p_{b}}{\partial \Lambda}+\frac{2}{\Lambda^{3}}\left(\frac{\partial p_{b}}{\partial \alpha}+\frac{2}{\Lambda^{2}} \frac{\partial \psi_{b}}{\partial \alpha}\right)\right] .\right.
$$

\subsection{Order- $F_{v}^{2}$ problem: determination of $\tilde{\boldsymbol{u}}_{20}$}

We now consider the order $F_{v}^{2}$. As explained previously, the second-order perturbations in $F_{v}$ are further expanded in $1 / \Lambda^{2}$ and only the zeroth-order solution in $1 / \Lambda^{2}$ $\left(\tilde{\boldsymbol{u}}_{20}, \tilde{p}_{20}, \tilde{\rho}_{20}\right)$ is determined. Therefore, to obtain $\left(\tilde{\boldsymbol{u}}_{20}, \tilde{p}_{20}, \tilde{\rho}_{20}\right)$, we need to take into account only the limits for $\Lambda \rightarrow \infty$ of the leading-order perturbation $\left(\tilde{\boldsymbol{u}}_{0}, \tilde{p}_{0}, \tilde{\rho}_{0}\right)$ and of the basic state $\boldsymbol{U}_{b}$. In this limit, the basic flow $\boldsymbol{U}_{b}$ in the neighbourhood of, for example, vortex 2 (i.e. at radial distance of order one from the centre of vortex 2) (figure 2) is at leading order an axisymmetric Lamb-Oseen vortex

$$
\boldsymbol{U}_{b}=\boldsymbol{U}_{a 2}+O\left(1 / \Lambda^{2}\right)
$$

since the velocity field $\boldsymbol{U}_{a 1}$, the adapted velocities $\boldsymbol{U}_{e 1} / \Lambda^{2}$ and $\boldsymbol{U}_{e 2} / \Lambda^{2}$ and the velocity due to the rotation of the reference frame are at most of order $1 / \Lambda^{2}$ and therefore negligible. In other words, the presence of vortex 1 can be neglected in computing the order- $F_{v}^{2} / \Lambda^{0}$ perturbation of vortex 2 and conversely, near vortex 1 , we can neglect the 
presence of vortex 2. Similarly, in the neighbourhood of vortex 2, the streamfunction (3.7) of the leading-order perturbation in the limit $\Lambda \rightarrow \infty$ approximates to

$$
\tilde{\psi}_{0}=\tilde{\psi}_{00}+O\left(1 / \Lambda^{2}\right)
$$

with

$$
\tilde{\psi}_{00}=-\frac{\Lambda}{2}\left[\delta \Lambda \frac{\partial \psi_{a 2}}{\partial x}(x-\Lambda, y)+\delta \alpha \frac{\partial \psi_{a 2}}{\partial y}(x-\Lambda, y)\right]
$$

where $\psi_{a 2}$ is the streamfunction associated with $\boldsymbol{U}_{a 2}$, i.e. $\boldsymbol{U}_{a 2}=-\nabla \times\left(\psi_{a 2} \boldsymbol{e}_{z}\right)$ and we recall that $(x, y)$ are the Cartesian coordinates centred on vortex 1 (figure 1). Thus, the perturbation of vortex 2 at zeroth order in $1 / \Lambda$ and $F_{v}$ consists of a small translation $\left(\beta_{2}=\frac{1}{2} \Lambda \delta \Lambda, \eta_{2}=\frac{1}{2} \Lambda \delta \alpha\right)$ in the $x$ - and $y$-directions (figure 2 ). The rotational invariance and the invariance linked to the parameter $\Lambda$ become equivalent in this limit to the translational invariances in the horizontal plane of a single axisymmetric vortex. The problem at order $F_{v}^{2} / \Lambda^{0}$ reduces therefore to the determination of a long-wavelength bending perturbation on a single axisymmetric vortex in a strongly stratified fluid. Note also that the background rotation is negligible since $\Omega=O\left(1 / \Lambda^{2}\right)$. Because the two basic vortices are symmetric and the perturbation considered here, (3.7), is also symmetric, it is sufficient to consider the bending perturbations $\left(\beta_{2}, \eta_{2}\right)$ on vortex 2 ; those on vortex $1\left(\beta_{1}, \eta_{1}\right)$ can be simply obtained by symmetry (figure 2$)$ :

$$
\beta_{1}=-\frac{\Lambda}{2} \delta \Lambda=-\beta_{2}, \quad \eta_{1}=-\frac{\Lambda}{2} \delta \alpha=-\eta_{2}
$$

Using the fact that $\psi_{a 2}=\int v_{\theta}(r) \mathrm{d} r, \tilde{\psi}_{00}$ can be simplified to

$$
\tilde{\psi}_{00}=-\left[\beta_{2} \cos \theta+\eta_{2} \sin \theta\right] v_{\theta}
$$

where we recall that $(r, \theta)$ are the cylindrical coordinates centred on vortex 2 (figure 1 ). The density perturbation $(3.14)$ reduces to

$$
\tilde{\rho}_{00}=\frac{v_{\theta}^{2}}{r}\left[\frac{\partial \beta_{2}}{\partial z} \cos \theta+\frac{\partial \eta_{2}}{\partial z} \sin \theta\right]
$$

while the vertical velocity (3.15) becomes

$$
\tilde{\boldsymbol{u}}_{z 00}=\frac{v_{\theta}^{3}}{r^{2}}\left[-\frac{\partial \beta_{2}}{\partial z} \sin \theta+\frac{\partial \eta_{2}}{\partial z} \cos \theta\right] .
$$

The physical meaning of equations (3.21) and (3.22) can be understood following a reasoning similar to the one given by Billant \& Chomaz $(2000 b)$. Because of the vertical modulation of $\eta_{2}$ and $\beta_{2}$, the low-pressure region located at the centre of the vortex is no longer aligned. The pressure anomalies are compensated by isopycnal distortions as described by equation (3.21). A physical representation is given in figure $3(a)$ for the case of a small unidirectional bend $\eta$ in the $y$-direction. These deformations of the isopycnal surfaces generate a vertical velocity field given by (3.22). As represented in figure 3(b), this vertical velocity can be interpreted physically as fluid particles in their merry-go-round rotation following the isopycnal surfaces in order to satisfy density conservation. Thus, they move up and down along the distortions of the isopycnal surfaces, producing a vertical velocity field. We shall see now that this vertical velocity induces in turn an horizontal flow at order $F_{v}^{2} / \Lambda^{0}$. 

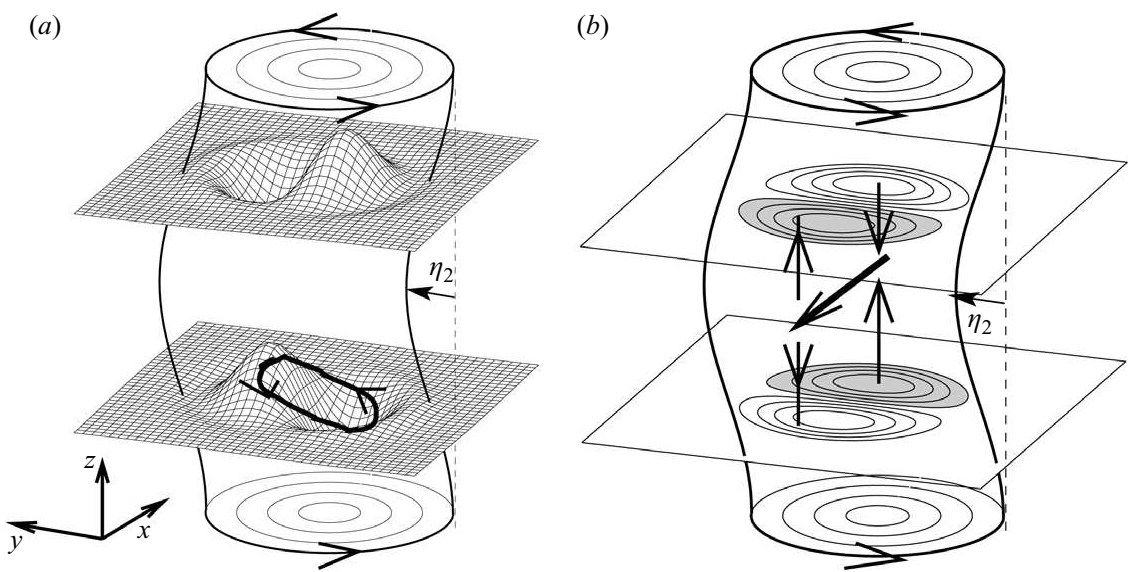

FiguRE 3. (a) Isopycnals given by (3.21) above and below a sinusoidal bend $\eta_{2}$ in the $y$-direction of a single axisymmetric vortex (the initial perturbation $\beta_{2}$ in the $x$-direction is assumed equal to 0 ). The amplitude of the deformation has been exaggerated. The trajectory of a particle is shown as a bold line on the lower surface. ( $b$ ) Contours of the vertical velocity $\tilde{u}_{z 00}$ given by (3.22). Shaded regions indicate upward motions. The vertical arrows show the direction of the vertical velocity. This vertical velocity field induces in the middle horizontal plane (not represented) a divergence at the front of the vortex and a convergence at the back. The larger horizontal arrow indicates the direction of the potential flow along the $x$-axis from the convergence to the divergence zones that is generated at order $F_{v}^{2}$ in order to satisfy mass conservation. The vertical velocity also stretches and squeezes the basic-state vertical vorticity in the middle horizontal plane: the vertical vorticity is increased at the front and decreased at the back in order to conserve potential vorticity. This effect also tends to displace the vortex in the direction of the horizontal arrow.

Using (3.20)-(3.22), the equations governing the perturbations at order $F_{v}^{2} / \Lambda^{0}$ in the neighbourhood of vortex 2 can be written:

$$
\begin{gathered}
\frac{\partial \tilde{\boldsymbol{u}}_{h 00}}{\partial t_{20}}+\left[\nabla_{h} \tilde{\boldsymbol{u}}_{h 20}\right] \cdot \boldsymbol{U}_{a 2}+\left[\nabla_{h} \boldsymbol{U}_{a 2}\right] \cdot \tilde{\boldsymbol{u}}_{h 20}=-\nabla_{h} \tilde{p}_{20} \\
\delta^{2}\left(\left[\nabla_{h} \tilde{\boldsymbol{u}}_{z 00}\right] \cdot \boldsymbol{U}_{a 2}\right)=-\frac{\partial \tilde{p}_{20}}{\partial z}-\tilde{\rho}_{20} \\
\nabla_{h} \cdot \tilde{\boldsymbol{u}}_{h 20}+\frac{\partial \tilde{u}_{z 00}}{\partial z}=0 \\
\frac{\partial \tilde{\rho}_{00}}{\partial t_{20}}+\left[\nabla_{h} \tilde{\rho}_{20}\right] \cdot \boldsymbol{U}_{a 2}-\tilde{u}_{z 20}=0
\end{gathered}
$$

where we recall that $\delta=F_{h} / F_{v}$. Note that a forcing term appears in the divergence equation $(3.23 c)$ due to the leading-order vertical velocity variation $\tilde{u}_{z 00}$. This implies that an horizontal velocity field is generated at order $F_{v}{ }^{2}$ in order to satisfy mass conservation.

In order to solve (3.23a) and (3.23c), we first decompose the horizontal velocity into rotational and potential components with a streamfunction $\psi_{20}$ and a potential function $\chi_{20}$ :

$$
\tilde{\boldsymbol{u}}_{h 20}=-\nabla \times\left(\psi_{20} \boldsymbol{e}_{z}\right)+\nabla_{h} \chi_{20} .
$$

By inserting this Helmholtz decomposition in the divergence equation (3.23c), one obtains

$$
\Delta_{h} \chi_{20}=-\frac{\partial \tilde{u}_{z 00}}{\partial z}
$$


with the boundary conditions that the velocity $\nabla_{h} \chi_{20}$ vanishes at infinity and that $\left(\partial^{2} \chi_{20} / \partial r^{2}\right)(0)=0$. The latter condition imposes that the velocity $\nabla_{h} \chi_{20}$ should be constant at $r=0$ but not necessarily zero since the vortex centre can move in the case of a displacement mode (see for example Leibovich, Brown \& Patel 1986). The solution is of the form

$$
\chi_{20}=\chi(r)\left(\cos \theta \frac{\partial^{2} \eta_{2}}{\partial z^{2}}-\sin \theta \frac{\partial^{2} \beta_{2}}{\partial z^{2}}\right)
$$

where the function $\chi$ can be determined analytically:

$$
\begin{aligned}
\chi= & \frac{\exp \left(-3 r^{2}\right)}{64 r^{3}}\left(3 r^{2}\left(-1+\exp \left(r^{2}\right)\right)^{2}-\left(-1+\exp \left(r^{2}\right)\right)^{3}\right)+\frac{3}{32 r} \ln \left(\frac{4}{3}\right) \\
& +\frac{3}{64 r}\left(\left(2+3 r^{2}\right) \operatorname{Ei}\left(-3 r^{2}\right)-4\left(1+r^{2}\right) \operatorname{Ei}\left(-2 r^{2}\right)+\left(2+r^{2}\right) \operatorname{Ei}\left(-r^{2}\right)\right)
\end{aligned}
$$

where $\mathrm{Ei}$ is the exponential integral function. The horizontal velocity $\nabla_{h} \chi_{20}$ is represented on figure $3(b)$ by an horizontal arrow. In order to satisfy mass conservation, this flow goes from the source (rear of the vortex) to the sink (front) created by the zeroth-order vertical velocity $\tilde{u}_{z 00}$.

We now take the curl of $(3.23 a)$ in order to eliminate the pressure and we insert the decomposition of the horizontal velocity (3.24):

$$
\mathrm{L}\left(\psi_{20}\right) \equiv \frac{\partial}{\partial \theta}\left(\frac{v_{\theta}}{r} \Delta_{h} \psi_{20}-\frac{1}{r} \psi_{20} \frac{\partial \omega}{\partial r}\right)=-\nabla_{h} \cdot\left(\omega \nabla_{h} \chi_{20}\right)+\frac{\partial \omega}{\partial r}\left[\sin \theta \frac{\partial \eta_{2}}{\partial t_{20}}+\cos \theta \frac{\partial \beta_{2}}{\partial t_{20}}\right] .
$$

The Fredholm alternative tells us that this equation has a solution only if the righthand side is orthogonal to the kernel of the adjoint $\mathrm{L}^{\dagger}$ of the linear operator $\mathrm{L}$. The adjoint $\mathrm{L}^{\dagger}$ for the scalar product, defined as

$$
\langle f \mid g\rangle=\int_{0}^{2 \pi} \int_{0}^{\infty} f g r \mathrm{~d} r \mathrm{~d} \theta
$$

is given in Appendix A. The functions $f=r \sin \theta, g=r \cos \theta$ and any function $h(r)$ are part of the kernel of $\mathrm{L}^{\dagger}$, i.e. $\mathrm{L}^{\dagger}(f)=\mathrm{L}^{\dagger}(g)=\mathrm{L}^{\dagger}(h)=0$. The right-hand side of (3.28) is always orthogonal to $h(r)$ but not to $g$ and $f$. The orthogonality condition with respect to $f$ is fulfilled only if

$$
\frac{\partial \eta_{2}}{\partial t_{20}}=D_{0} \frac{\partial^{2} \beta_{2}}{\partial z^{2}}
$$

and the one with respect to $g$ is satisfied only if

$$
\frac{\partial \beta_{2}}{\partial t_{20}}=-D_{0} \frac{\partial^{2} \eta_{2}}{\partial z^{2}}
$$

where $D_{0}$ is a constant defined by

$$
D_{0}=-\frac{\left\langle\nabla_{h} \cdot\left(\omega \nabla_{h}(\chi \sin \theta)\right) \mid r \sin \theta\right\rangle}{\left\langle\frac{\partial \omega}{\partial r} \sin \theta \mid r \sin \theta\right\rangle} .
$$

Therefore, the above two solvability conditions give the evolution equations of $\left(\eta_{2}\right.$, $\beta_{2}$ ) over the slow time scale $t_{20}$. 
After some simplifications, we obtain

$$
D_{0}=\int_{0}^{\infty} r \frac{\partial \omega}{\partial r} \chi \mathrm{d} r
$$

which can be calculated exactly from the exact expression (3.27) of $\chi$ :

$$
D_{0}=\frac{7}{8} \ln 2-\frac{9}{16} \ln 3=-0,0115 \ldots
$$

Note that if we insert in (3.30)-(3.31) perturbations of the form

$$
\left(\begin{array}{c}
\eta_{2} \\
\beta_{2}
\end{array}\right)=\left(\begin{array}{c}
\tilde{\eta}_{2} \\
\tilde{\beta}_{2}
\end{array}\right) \mathrm{e}^{\sigma t_{20}+\mathrm{i} k z},
$$

a purely imaginary growth rate $\sigma$ is obtained:

$$
\sigma^{2}=-D_{0}^{2} k^{2}
$$

In other words, a single axisymmetric vortex in a strongly stratified fluid is neutral with respect to small translations in the long-wavelength limit.

The bending deformation will thus rotate around the vortex centre without any growth. Note that the direction of rotation of the bending perturbation is the same as the fluid inside the vortex since $D_{0}$ is negative. This can be deduced from (3.30)-(3.31) and from figure 3 where a bending perturbation such that $\partial^{2} \eta_{2} / \partial z^{2}$ is negative and $\beta_{2}=0$ has been imposed initially. According to the solvability condition (3.31), this implies a decrease of $\beta_{2}$ as time evolves because $D_{0}$ is negative. Thus, the bending deformation starts to move in the negative $x$-direction so that it will have the same direction of rotation as the basic vortex. This direction of rotation can also be directly deduced from the potential flow $\nabla_{h} \chi_{20}$ generated at $O\left(F_{v}^{2}\right)$, which is directed from the source to the sink (as shown by the horizontal arrow on figure $3 b$ ). The effect of the stretching and squeezing of the basic potential vorticity by the vertical velocity (contained in (3.28)) is also in the same direction. In sharp contrast, it is interesting to remark that the direction of rotation of a bending deformation for an isolated vortex in homogeneous fluid is opposite to the basic vortex (Batchelor 1967).

Once the stability conditions (3.30)-(3.31) are satisfied, $\psi_{20}$ can be determined from (3.28). This is carried out in Appendix B. For large radius $r$, the potential $\chi_{20}$ and the streamfunction $\psi_{20}$ are proportional to $1 / r$, implying that the associated velocity varies, proportional to $1 / r^{2}$. Therefore, the second-order perturbation, $O\left(F_{v}^{2} / \Lambda^{0}\right)$, of one vortex has an $O\left(F_{v}^{2} / \Lambda^{2}\right)$ effect on the other vortex which is separated by distance $\Lambda$. This effect can be neglected at the level of approximation considered in the present paper, justifying a posteriori that it was fully legitimate to consider an isolated vortex to determine the second-order perturbation, $O\left(F_{v}^{2} / \Lambda^{0}\right)$.

\section{Stability analysis}

The slow time scales $t_{02}$ and $t_{20}$ are now re-expressed in terms of the fast time $t$ :

$$
\frac{\partial}{\partial t}=\frac{1}{\Lambda^{2}} \frac{\partial}{\partial t_{02}}+F_{v}{ }^{2} \frac{\partial}{\partial t_{20}}+\cdots .
$$

Then, the equations (3.11)-(3.12) and (3.30)-(3.31) can be combined to give

$$
\begin{gathered}
\frac{\partial \delta \alpha}{\partial t}=-2 \frac{\delta \Lambda}{\Lambda^{2}}+D_{0} F_{h}^{2} \frac{\partial^{2} \delta \Lambda}{\partial \tilde{z}^{2}}+O\left(F_{h}^{4}, \frac{F_{h}^{2}}{\Lambda^{2}}\right), \\
\frac{\partial \delta \Lambda}{\partial t}=-D_{0} F_{h}^{2} \frac{\partial^{2} \delta \alpha}{\partial \tilde{z}^{2}}+O\left(F_{h}^{4}, \frac{F_{h}^{2}}{\Lambda^{2}}\right),
\end{gathered}
$$




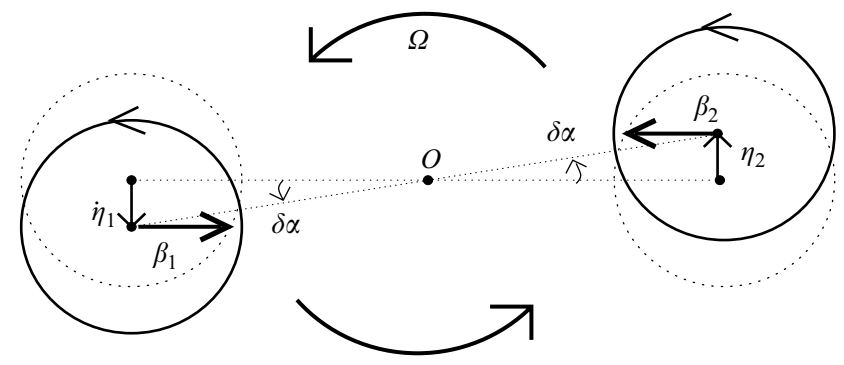

FIGURE 4. Sketch of the mechanism of the zigzag instability: the vortex pair (dotted line) is perturbed by a small rotation $\delta \alpha$ modulated vertically. The perturbed vortex pair is shown by solid lines. Since $\delta \alpha$ is small, the perturbation is equivalent to a $y$-translation of the two vortices in opposite directions $\left(\eta_{1}<0, \eta_{2}>0\right)$. Three-dimensional effects then displace the two vortices along the $x$-axis such that the two vortices move closer $\left(\beta_{1}>0, \beta_{2}<0\right)$. Thus the rotation speed and the initial perturbation $\delta \alpha$ increases.

where we have used the relation $\left(\beta_{2}=\frac{1}{2} \Lambda \delta \Lambda, \eta_{2}=\frac{1}{2} \Lambda \delta \alpha\right)$ and where the vertical coordinate $\tilde{z}$ is now non-dimensionalized by the vortex radius so that $\tilde{z}=\left(F_{h} / F_{v}\right) z$ where $z$ is the previous vertical coordinate non-dimensionalized by $L_{v}$. For this reason, the horizontal Froude number appears in (4.2)-(4.3) instead of the vertical Froude number.

Inserting a perturbation of the form $(\delta \alpha, \delta \Lambda)=\left(\delta \alpha_{0}, \delta \Lambda_{0}\right) \mathrm{e}^{\sigma t+\mathrm{i} k_{z} z}$ yields

$$
\sigma^{2}=-\frac{2}{\Lambda^{2}} D_{0}\left(F_{h} k_{z}\right)^{2}-D_{0}^{2}\left(F_{h} k_{z}\right)^{4}+O\left(F_{h}^{6}, \frac{F_{h}^{2}}{\Lambda^{4}}, \frac{F_{h}^{4}}{\Lambda^{2}}\right) .
$$

Quite strikingly, we see in equation (4.4) that there is an instability in the smallwavenumber limit since $D_{0}$ is negative. The higher-order term $O\left(F_{h}^{4} k_{z}^{4}\right)$ is negative however and thus stabilizing. The dispersion is self-similar with respect to the variable $F_{h} k_{z}$ in agreement with Billant \& Chomaz (2001). The maximum amplification $\sigma_{m}=$ $1 / \Lambda^{2}$ (in dimensional form $\Gamma / \pi b^{2}$ ) is equal to twice the external strain $S=1 / 2 \Lambda^{2}$ independently of the Froude number $F_{h}$. It is reached for a finite wavenumber $k_{m}=\sqrt{-1 / D_{0}}\left(1 / F_{h} \Lambda\right)$ proportional to $1 / F_{h} \Lambda$, meaning that the dimensional wavelength $\lambda$ of the instability is proportional to the buoyancy length $\Gamma / \pi a N$ multiplied by the ratio $b / a: \lambda \propto F_{h} b$. This scaling implies that $k_{z} F_{h}=O\left(F_{v}\right)=O(1 / \Lambda)$, confirming that it was legitimate to neglect $O\left(F_{v}^{2} / \Lambda^{2}\right)$ in addition to $O\left(F_{v}^{4}\right)$ in the expansion (3.3).

The physical mechanism of the instability can be easily understood. As sketched in figure 4, a small perturbation of the orientation angle of the vortex pair $(\delta \alpha>0)$ implies a negative $y$-translation $\eta_{1}=-\delta \alpha \frac{1}{2} \Lambda$ of vortex 1 and a positive $y$-translation $\eta_{2}=\delta \alpha \frac{1}{2} \Lambda$ of vortex 2 . In the two-dimensional case, such a perturbation is neutral because of the rotational invariance. However, according to (3.30)-(3.31), if $\eta_{1}$ and $\eta_{2}$ vary slowly with $z$, a positive (negative) translation in the $y$-direction of a vortex rotating in the positive sense generates at order $F_{h}{ }^{2} k_{z}^{2}$ a self-induced displacement in the negative (positive) $x$-direction. Therefore, $\beta_{2}<0$ and $\beta_{1}>0$ (figure 4). The two vortices thus move closer $(\delta \Lambda<0)$ so that they will rotate faster around each other, amplifying the initial perturbation $\delta \alpha$.

Conversely, a negative perturbation of the orientation of the vortex pair $(\delta \alpha<0)$ implies an increase of the distance between the vortices $(\delta \Lambda>0)$. They will therefore rotate slower, increasing the initial phase shift $|\delta \alpha|$. The result of the zigzag instability 


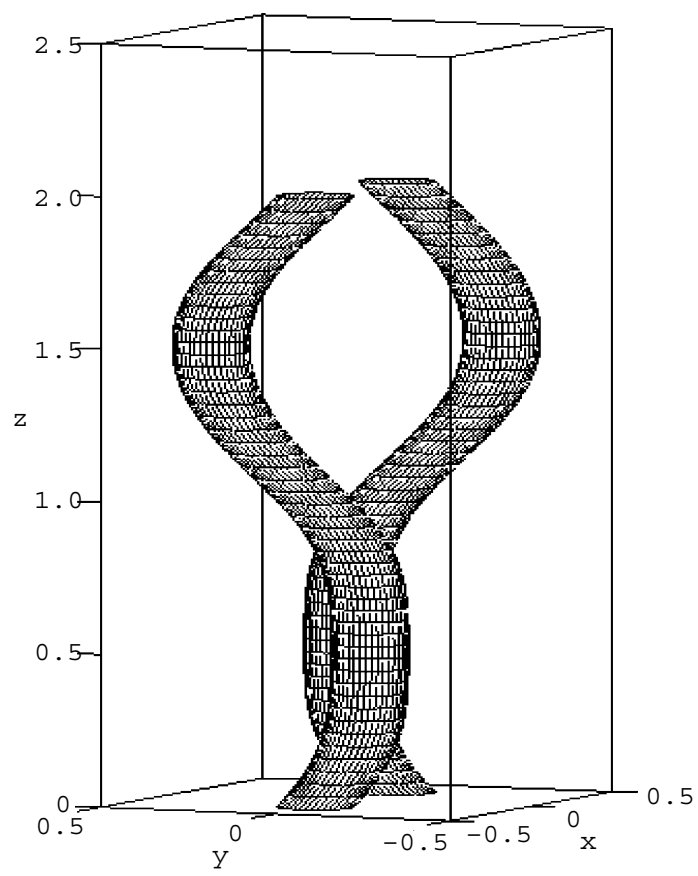

FIGURE 5. Shape of the bending deformations induced by the zigzag instability on the co-rotating vortex pair. This picture is schematic since the linear perturbation has been added to the basic state with an arbitrary $O(1)$ amplitude in order to make the deformation visible.

is to move the two vortices closer together or furthers apart every half a wavelength as sketched on figure 5 .

From this explanation and from (4.4), we can see that the fact that $D_{0}$ is negative is crucial for the existence of the instability. If $D_{0}$ were positive, the co-rotating vortex pair would not be unstable to such perturbations because the self-induced displacements would be in opposite directions (i.e. $\beta_{2}>0$ and $\beta_{1}<0$ ). As mentioned above, this is the case in homogeneous fluids, which explains why this instability of a co-rotating vortex pair is specific to strongly stratified fluids and does not exist in homogeneous fluids as shown by Jimenez (1975).

\section{Comparison between theoretical and numerical results}

In this section, we compare the numerical results of the linear stability analysis of the zigzag instability (Otheguy et al. 2006) and the present asymptotic theory.

\subsection{Growth rate as a function of the wavenumber and Froude number}

Figure 6(a) compares the asymptotic growth rate of the zigzag instability (4.4) as a function of the wavenumber $k_{z}$ with the growth rate obtained in the numerical linear stability analysis for several horizontal Froude numbers, for a high Reynolds number $R e=16000$ and a single ratio $1 / \Lambda=0.15$. The asymptotic and numerical results are in good agreement for all $F_{h}$ even for large $F_{h}$ up to 1.7. The agreement is better for small wavenumber for two reasons: the viscous damping, not taken into account in the theoretical stability analysis, increases with $k_{z}$ and the long-wavelength approximation used in the asymptotic analysis is better for small $k_{z}$. 
(a)

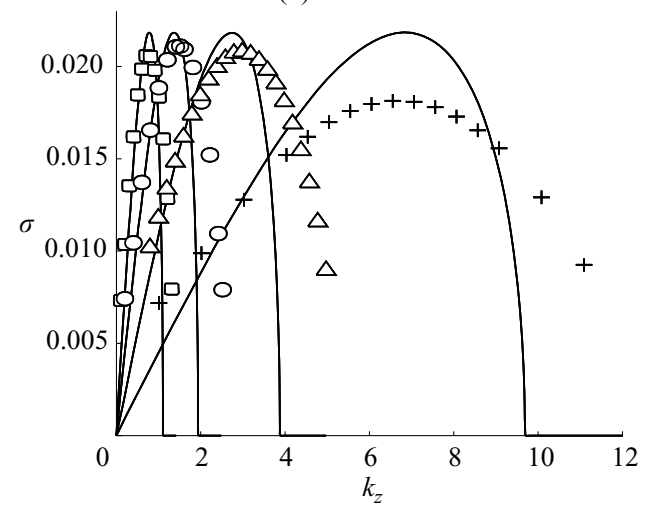

(b)

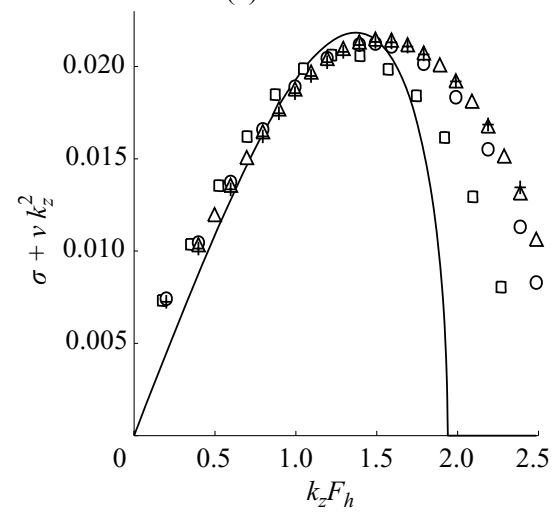

FIgURE 6. (a) Comparison between the theoretical growth rate (4.4) (curves) and the growth rate obtained numerically as a function of the vertical wavenumber $k_{z}$ for different horizontal Froude numbers: $F_{h}=1.7,1,0.5,0.2$ (from right to left). $(b)$ Comparison between the theoretical growth rate (4.4) (curve) and the numerical growth rates compensated for the vertical viscous damping (symbols) as a function of the vertical wavenumber rescaled by the horizontal Froude number $k_{z} F_{h}$. The symbols represent the numerical growth rates for $F_{h}=1.7$ $(\square), F_{h}=1(\bigcirc), F_{h}=0.5(\triangle)$ and $F_{h}=0.2(+)$, the Reynolds number being $R e=16000$ and the ratio $1 / \Lambda=0.15$.

In order to take into account the viscous effects, figure $6(b)$ compares the asymptotic growth rate as a function of $k_{z} F_{h}$ to the growth rate obtained numerically and compensated for the vertical viscous damping $\left(\sigma+\nu k_{z}^{2}\right)$. The asymptotic analysis predicts correctly the compensated maximum growth rate. The agreement of the evolution of the growth rate as a function of $k_{z} F_{h}$ is remarkable in view of the low-order asymptotics for $k_{z} F_{h} \lesssim k_{z m} F_{h}$, where $k_{z m}$ is the most unstable wavenumber. As stated previously, the asymptotic and numerical growth rates depart for large $k_{z} F_{h}$, owing to the small-wavenumber assumption $k_{z} F_{h}=O\left(F_{v}\right) \ll 1$ used in the asymptotic analysis.

\subsection{Maximum growth rate and most amplified wavenumber as a function of $1 / \Lambda$}

In this subsection, we further investigate the effect of the ratio $1 / \Lambda$, characterizing the basic state, on the maximum growth rate and on the most unstable wavenumber for a given Froude number $F_{h}=1$. As seen in figure 7, the agreement between the asymptotic and numerical results is very good for small $1 / \Lambda$ both for the maximum unstable growth rate (figure $7 a$ ) and for the most unstable wavenumber (figure $7 b$ ). This confirms that the maximum growth rate of the zigzag instability is twice the strain $S=1 / 2 \Lambda^{2}$ and that the most amplified wavenumber is inversely proportional to $F_{h} \Lambda$. As the asymptotic analysis is valid only for small $1 / \Lambda$, it is not surprising that the numeric and the asymptotic results start diverging when $1 / \Lambda$ increases. The maximum growth rate obtained numerically tends to be smaller than the theoretical prediction while the most amplified wavenumber is larger than the theoretical prediction.

\subsection{Shape of the eigenmode as a function of $k_{z} F_{h}$}

The vertical vorticity of the eigenmode of the zigzag instability can be obtained at leading order by taking the horizontal Laplacian of (3.7) in the limit $\Lambda \rightarrow \infty$ and $F_{v}=0$ using the phase equations (4.2)-(4.3) and the dispersion relation (4.4) which 

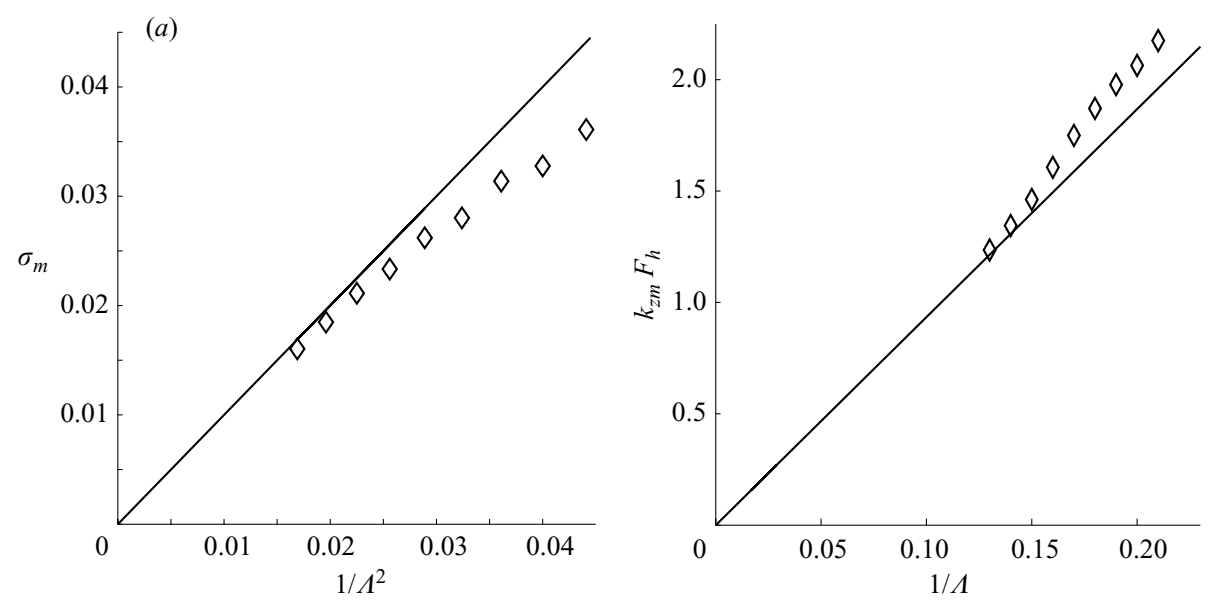

FiguRE 7. Comparison of $(a)$ the maximum growth rate against $1 / \Lambda^{2}$ and $(b)$ of the most amplified wavenumber against $1 / \Lambda$, obtained numerically for $F_{h}=1$ and $R e=16000$ (diamonds) and asymptotically (line).

couples $\delta \alpha$ and $\delta \Lambda$ :

$$
\begin{aligned}
\Delta_{h} \tilde{\psi}_{0}= & -\frac{\Lambda}{2} \delta \alpha\left[\frac{\partial}{\partial y}-F_{h} k_{z} \sqrt{\frac{-D_{0}}{2 / \Lambda^{2}+D_{0}\left(F_{h} k_{z}\right)^{2}}} \frac{\partial}{\partial x}\right] \mathrm{e}^{-(x-\Lambda)^{2}-y^{2}} \\
& +\frac{\Lambda}{2} \delta \alpha\left[\frac{\partial}{\partial y}-F_{h} k_{z} \sqrt{\frac{-D_{0}}{2 / \Lambda^{2}+D_{0}\left(F_{h} k_{z}\right)^{2}}} \frac{\partial}{\partial x}\right] \mathrm{e}^{-x^{2}-y^{2}} .
\end{aligned}
$$

As already mentioned in $\S 4$, the perturbation described by equation (5.1) consists of a symmetric translation of each vortex along both the $x$ - and $y$-directions. The angle $\zeta$ between the direction of translation of each vortex and the negative $x$-axis (figure 8 ) is given by the ratio of the coefficients in front of the derivatives $\partial / \partial y$ and $-\partial / \partial x$ in equation (5.1):

$$
\tan (\zeta)=\sqrt{\frac{2 / \Lambda^{2}+D_{0}\left(F_{h} k_{z}\right)^{2}}{-D_{0}}} \frac{1}{F_{h} k_{z}} .
$$

At small $F_{h} k_{z}, \zeta$ goes to $\pi / 2$ and the translation is along the $y$-axis for both vortices but in opposite directions for vortex 1 and vortex 2 . The perturbation is thus equivalent to a rotation of the vortex pair. When $F_{h} k_{z}$ increases, there is an additional small component of translation along the $x$-axis such that the two vortices move closer to or away from each other. Figure 8 shows that the eigenmode given by (5.1) is in very good agreement with the vertical vorticity of the eigenmode obtained numerically for different vertical wavenumbers and horizontal Froude numbers. The dipole shape of the perturbation observed inside each vortex of the basic state corresponds to a translation in an oblique direction whose angle $\zeta$ decreases from $\pi / 2$ similarly in the numerical and asymptotic eigenmodes when the product $k_{z} F_{h}$ increases. Figure 9 compares (further) the angle $\zeta$ predicted theoretically to the values measured for the eigenmodes obtained numerically. The agreement is excellent except at large $k_{z} F_{h}$ for which the assumption $k_{z} F_{h} \ll 1$ used in the asymptotic theory is no longer valid. 


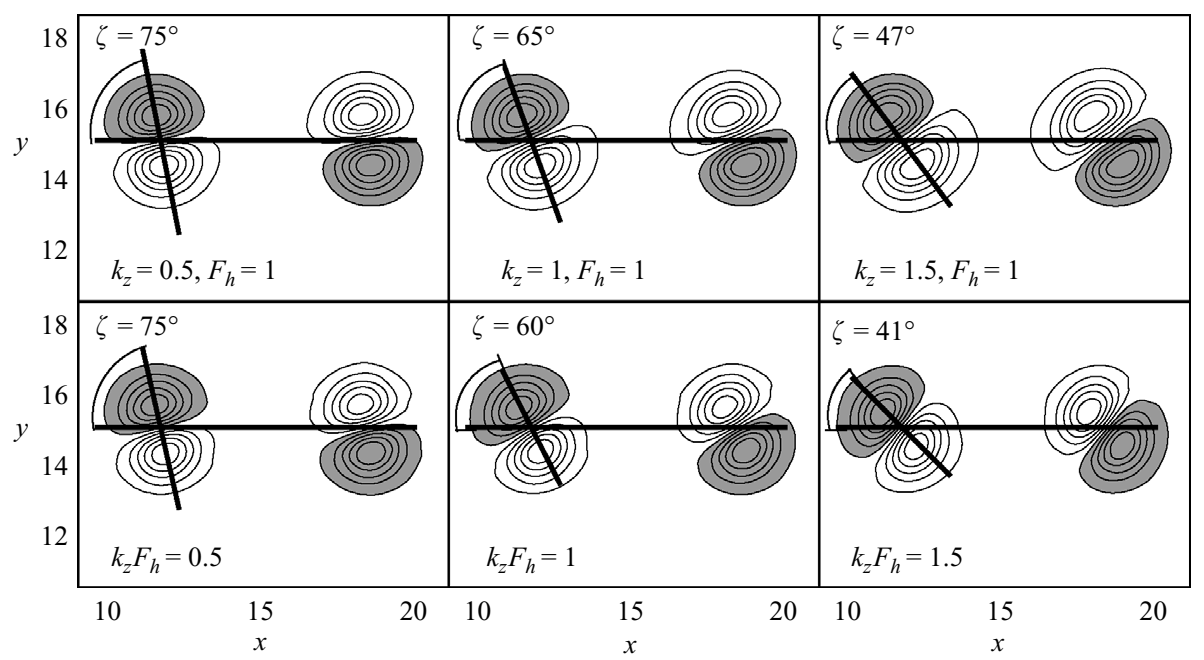

FIGURE 8. Comparison between the vertical vorticity of the eigenmodes obtained numerically (top row) and the asymptotic eigenmodes (bottom row) for various products $k_{z} F_{h}$. The value of the angle $\zeta$ between the negative $x$-axis and the line joining the extrema of vorticity of the dipole pattern is also shown. The numerical simulations correspond to $R e=500 ; 1 / \Lambda=0.15$ (see Otheguy et al., 2006 for further details).

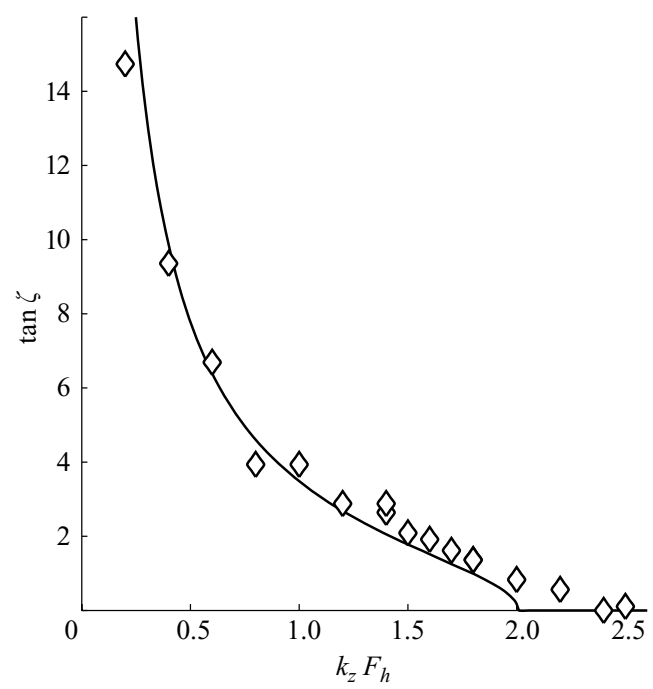

Figure 9. Comparison between the orientation of the dipole pattern of the eigenmodes (line joining the minimum and maximum vertical vorticity of the dipole pattern) obtained asymptotically (curve) and numerically (diamonds) as a function of $k_{z} F_{h}$. The numerical simulations correspond to $R e=16000 ; 1 / \Lambda=0.15$.

\section{Summary and conclusions}

In this paper, we have performed a linear stability analysis of two co-rotating Gaussian vortices in a strongly stratified fluid $\left(F_{h} \ll 1\right)$ using a multiple-scale expansion with respect to two expansion parameters: the vertical Froude number $F_{v}$ and the inverse of the separation ratio $1 / \Lambda=a / b$. This analysis fully explains 
qualitatively and quantitatively the zigzag instability found numerically by Otheguy et al. (2006).

This instability is specific to strongly stratified fluids and does not exist in homogeneous fluids (Jimenez 1975).

The zigzag instability is triggered by the coupling between the long-wavelength bending waves on each vortex and the basic strain field that each vortex exerts on its companion. If the co-rotating vortex pair is twisted in vertically with respect to the rotation centre, the bending waves on each vortex tend to bring the two vortices closer together (farther a part) at the vertical location where the angle of the vortex pair is increased (decreased). Thus, the vortices will rotate faster and slower alternately vertically, increasing the initial twist in an unstable manner.

The asymptotic analysis is in excellent quantitative agreement with the numerical linear stability analysis (Otheguy et al. 2006). The dimensional growth rate of the zigzag instability is twice the strain rate $\left(S=\Gamma / 2 \pi b^{2}\right)$ generated by each vortex on the other vortex, independently of the stratification. The most unstable wavelength $\lambda$ scales as the buoyancy length $L_{B}=\Gamma / \pi a N$ multiplied by the ratio $b / a, \lambda \propto F_{h} b$. In other words, the most unstable vertical scale is inversely proportional to the BruntVäisälä frequency and increases with the separation distance between the vortices.

The zigzag instability has been shown to affect co- and counter-rotating vortex pairs of equal strength. We have shown here that it comes from a generic mechanism: the coupling of the slow bending waves of two vortices through the strain field; it is thus likely that it will affect any flow consisting of several vortices in a strongly stratified fluid so it could explain the emergence of layers observed in complex flows (Park et al. 1994; Holford \& Linden 1999; Waite \& Bartello 2004; Lindborg 2006). Lindborg (2006) has recently shown that a strongly stratified turbulent flow that is initially vertically coherent spontaneously develops horizontal layers. Their thickness scales with the horizontal Froude number, in full agreement with the present results for the instability wavelength and the scaling law of Billant \& Chomaz (2001).

However, in order to go beyond linear effects and explain the characterics of fully developed layers, the nonlinear behaviour of the zigzag instability will be investigated in a forthcoming paper.

\section{Appendix A. Adjoint operator}

The adjoint operator $\mathrm{L}^{\dagger}$ is defined by

$$
\langle f \mid L(g)\rangle=\left\langle L^{\dagger}(f) \mid g\right\rangle
$$

where the scalar product is defined in (3.29) and the operator $L$ in (3.28). We obtain after several integrations by parts:

$$
\mathrm{L}^{\dagger}(f)=2 \frac{\partial f}{\partial \theta}\left(\frac{v_{\theta}^{\prime}}{r^{2}}-\frac{v_{\theta}}{r^{3}}\right)-\frac{\partial^{3} f}{\partial \theta^{3}} \frac{v_{\theta}}{r^{3}}+\frac{\partial^{2} f}{\partial \theta \partial r}\left(\frac{v_{\theta}}{r^{2}}-2 \frac{v_{\theta}^{\prime}}{r}\right)-\frac{\partial^{3} f}{\partial^{2} r \partial \theta} \frac{v_{\theta}}{r}
$$

where $f$ is assumed to be $2 \pi$ periodic, and $\partial f / \partial \theta$ and $\partial^{2} f / \partial \theta \partial r$ are both assumed to be finite when $r$ goes to zero and to infinity.

\section{Appendix B. Determination of $\psi_{20}$}

Equation (3.28) suggests writing $\psi_{20}$ in the form

$$
\psi_{20}=\psi(r)\left(\cos \theta \frac{\partial^{2} \beta_{2}}{\partial z^{2}}+\sin \theta \frac{\partial^{2} \eta_{2}}{\partial z^{2}}\right) .
$$


The function $\psi$ is then determined numerically as the solution of

$$
\frac{v_{\theta}}{r} \psi^{\prime \prime}+\frac{v_{\theta}}{r^{2}} \psi^{\prime}-\left(\frac{v_{\theta}^{\prime}}{r^{2}}+\frac{v_{\theta}^{\prime \prime}}{r}\right) \psi=\left(-\chi^{\prime}-D_{0}\right) \omega^{\prime}+\omega \frac{v_{\theta}{ }^{3}}{r^{2}}
$$

associated with the boundary conditions $(\partial / \partial r)(\psi / r)(0)=0$ and $\psi^{\prime}(r) \rightarrow 0$ when $r$ goes to infinity.

\section{REFERENCES}

Batchelor, G. K. 1967 An Introduction to Fluid Dynamics. Cambridge University Press.

Billant, P. \& Chomaz, J.-M. $2000 a$ Experimental evidence for a new instability of a vertical columnar vortex pair in a strongly stratified fluid. J. Fluid Mech. 418, 167-188.

Billant, P. \& Chomaz, J.-M. $2000 b$ Theoretical analysis of the zigzag instability of a vertical columnar vortex pair in a strongly stratified fluid. J. Fluid Mech. 419, 29-63.

Billant, P. \& Chomaz, J.-M. 2000c Three-dimensional stability of a vertical columnar vortex pair in a stratified fluid. J. Fluid Mech. 419, 65-91.

Billant, P. \& Chomaz, J.-M. 2001 Self-similarity of strongly stratified inviscid flows. Phys. Fluids 13, 1645-1651.

Bonnier, M., Eiff, O. \& Bonneton, P. 2000 On the density structure of far-wake vortices in a stratified fluid. Dyn. Atmos. Oceans 31, 117-137.

Eloy, C. \& Le Dizès, S. 1999 Three-dimensional instability of Burgers and Lamb-Oseen vortices in a strain field. J. Fluid Mech. 378, 145-166.

Fincham, A. M., Maxworthy, T. \& Spedding, G. R. 1996 Energy dissipation and vortex structure in freely decaying, stratified grid turbulence. Dyn. Atmos. Oceans 23, 155-169.

Godeferd, F. S. \& Staquet, C. 2003 Statistical modelling and direct numerical simulations of decaying stably stratified turbulence. Part 2. Large-scale and small-scale anisotropy. J. Fluid Mech. 486, 115-159.

Godoy-Diana, R., Chomaz, J. M. \& Billant, P. 2004 Vertical length scale selection for pancake vortices in strongly stratified viscous fluids. J. Fluid Mech. 504, 229-238.

Herring, J. R. \& MÉtAis, O. 1989 Numerical experiments in forced stably stratified turbulence. J. Fluid Mech. 202, 97-115.

Holford, J. M. \& Linden, P. F. 1999 Turbulent mixing in a stratified fluid. Dyn. Atmos. Oceans 30, $173-198$.

JimeneZ, J. 1975 Stability of a pair of co-rotating vortices. Phys. Fluids 18, 1580-1582.

Lamb, H. 1932 Hydrodynamics, 6th edn. Cambridge University Press.

LE Dizès, S. \& LAPORTE, F. 2002 Theoretical predictions for the elliptical instability in a two-vortex flow. J. Fluid Mech. 471, 169-201.

Leibovich, S., Brown, S. N. \& PAtel, Y. 1986 Bending waves on inviscid columnar vortices. J. Fluid Mech. 173, 595-624.

LiLly, D. K. 1983 Stratified turbulence and the mesoscale variability of the atmosphere. J. Atmos. Sci. 40, 749-761.

LindBORG, E. 2006 The energy cascade in a strongly stratified fluid. J. Fluid Mech. 550, $207-242$.

Moffatt, H. K., Kida, S. \& OhKitani, K. 1994 Stretched vortices-the sinews of turbulence-large reynolds number asymptotics. J. Fluid Mech. 259, 241-264.

Moore, D. W. \& Saffman, P. G. 1975 The instability of a straight vortex filament in a strain field. Proc. R. Soc. Lond. 346, 413-425.

Otheguy, P., Chomaz, J.-M. \& Billant, P. 2006 Elliptic and zigzag instabilities on co-rotating vertical vortices in a stratified fluid. J. Fluid Mech. 553, 253-272.

Park, Y.-G., Whitehead, J. A. \& Gnanadeskian, A. 1994 Turbulent mixing in stratified fluids: layer formation and energetics. J. Fluid Mech. 279, 279-311.

Praud, O., Fincham, A. M. \& Sommeria, J. 2005 Decaying grid turbulence in a strongly stratified fluid. J. Fluid Mech. 522, 1-33.

Riley, J. J. \& Lelong, M.-P. 2000 Fluid motions in the presence of strong stable stratification. Annu. Rev. Fluid Mech. 32, 617-657. 
Riley, J. J., Metcalfe, W. \& Weissman, M. A. 1981 Direct numerical simulations of homogeneous turbulence in density-stratified fluids. Proc. AIP Conf. Nonlinear Properties of Internal Waves (ed. B. J. West), pp. 79-112.

Saffman, P. G. 1992 Vortex Dynamics. Cambridge University Press.

Spedding, G. R., Browand, F. K. \& Fincham, A. M. 1996 Turbulence, similarity scaling and vortex geometry in the wake of a sphere in a stably-stratified fluid. J. Fluid Mech. 314, 53-103.

Waite, M. L. \& Bartello, P. 2004 Stratified turbulence dominated by vortical motion. J. Fluid Mech. 517, 281-308. 